\title{
Defective Areas Identification in Aircraft Components by Bivariate EMD Analysis of Ultrasound Signals
}

\author{
Marco Leo ${ }^{1}$, David Looney ${ }^{2}$, Tiziana D'Orazio ${ }^{1}$, and Danilo P. Mandic ${ }^{2}$ \\ 1 Institute of Intelligent Systems for Automation, \\ Italian National Research Council, Bari, Italy \\ \{leo, dorazio\}@ba.issia.cnr.it \\ 2 Department of Electrical and Electronic Engineering, \\ Imperial College of Science, Technology and Medicine, London, UK \\ \{david.looney06,d.mandic\}@imperial.ac.uk
}

\begin{abstract}
In recent years many alternative methodologies and techniques have been proposed to perform non-destructive inspection and maintenance operations of moving structures. In particular, ultrasonic techniques have shown to be very promising for automatic inspection systems. From the literature, it is evident that the neural paradigms are considered, by now, the best choice to automatically classify ultrasound data. At the same time the most appropriate pre-processing technique is still undecided. The aim of this paper is to propose a new and innovative data pre-processing technique that allows the analysis of the ultrasonic data by a complex extension of the Empirical Mode Decomposition (EMD). Experimental tests aiming to detect defective areas in aircraft components are reported and a comparison with classical approaches based on data normalization or wavelet decomposition is also provided.
\end{abstract}

\section{Introduction}

The challenge of guaranteeing reliable and efficient safety checks for engineering structures has received much attention in recent years in many industrial contexts. This is of crucial importance in the case of moving structures (such as with transportation vehicles or rotating machinery) and in the case of aircraft inspection and maintenance. Traditionally inspection and maintenance operation are performed by trained human operators but, unfortunately, this approach does not ensure an adequate reliability level and, at the same time, it requires prohibitive amounts of time and high costs. In addition, humans cannot detect cracks or any other irregularities in the structure components which are not visible to the naked eye. To face the above problems, many alternative methodologies and techniques have been proposed to perform non-destructive inspection and maintenance operations. These are based on the analysis of different signals such as ultrasonics, acoustic emissions, thermography, laser ultrasonics, X-radiography, eddy currents, shearography, and low-frequency methods [1].

F. Schwenker and N. El Gayar (Eds.): ANNPR 2010, LNAI 5998, pp. $219-230,2010$.

(C) Springer-Verlag Berlin Heidelberg 2010 
In particular, in the last decade, ultrasonic techniques have shown to be very promising for non-destructive inspection and control, becoming an effective alternative to such traditional and well studied approaches such as thermography, eddy current and shearography.

Major works of literature describing ultrasound based techniques for inspection and evaluation purposes can be conveniently clustered into two categories. The first category concentrates on the study of the data acquisition and manipulation processes in order to prove the relationship between data and structural defects or composition of the material. The second category concentrates instead on the a posteriori analysis of the ultrasound data in order to (fully or partially) refer to some computational algorithm the automatic recognition of material composition, operative conditions, presence of defects and so on. Most of the works in the literature belong to the first category [12 [4] 10].

Works belonging to the second category are the least developed of the two and, moreover, their level of inspection reliability, is still inadequate, especially for those sectors (namely transportation) where an error can have serious health and safety consequences. Almost all the works in this category proposed a well-known framework based on some pre-processing technique followed by a classifier able to recognize the patterns in the data. For example in [7, the wavelet transform was used in conjunction with an artificial neural network to distinguish the ultrasonic flaw echoes from those scattered by micro-structures. A quite similar approach was introduced in 11] which addressed the problem of pipe inspection by ultrasonic guided waves.

The automatic detection of internal defects in composite materials with nondestructive techniques based on ultrasonic techniques was addressed also in [14.

In 8 the authors addressed the flaw detection problem by using a radial basis function neural network and they tried to demonstrate that a neural based approach overcame the classical threshold based for flaw detection problems. The author's idea in 13 was, instead, to cluster the signals in the similarity space (using the Kohonen Self organizing Algorithm to cluster datasets in an unsupervised manner) and then to use this result in order to distinguish between signals corresponding respectively to non defect, flat defects (cracks), and volumetric defects. A high resolution pursuit based signal processing method (an enhanced version of the matching pursuit algorithm that provides high time-resolution time-frequency representations and resolves closely time-spaced features) was proposed in [5] for detecting flaws close to the surface of strongly scattering materials, such as steel and composites, in NDT applications. In 6] an approach to non-destructive pipeline testing using ultrasonic imaging was proposed.

Finally, in [9], an evaluation of various types and configurations of neural networks developed for the purpose of assisting in accurate flaw detection in steel plates was illustrated.

Therefore, from the literature, it is evident that the neural paradigms are considered, by now, the best choice to classify ultrasound data in an automatic inspection system. At the same time the most appropriate pre-processing technique is still undecided. The wavelet based approaches seem to be the most 
promising ones but, considering that in this area an error could waste time, money and even endanger someone's life, further efforts have to be done in order to increase reliability.

The aim of this paper is then to propose a new and innovative data preprocessing technique to explore the pattern embedded in the data. A comparison with classical approaches based on data normalization or wavelet decomposition is also provided. In the proposed pre-processing procedure the complex valued Fourier coefficients of the ultrasound signals are decomposed by using Empirical Mode Decomposition (EMD) [16] into a set of oscillation modes or IMFs (intrinsic mode functions). In this way, the phase information is defined locally for both the real and imaginary components of the decomposition. This facilitates the detection of the temporal locking of phase information between the components, known as phase synchrony. The existence of phase synchrony between a pair of components has been used to characterise shared signal dynamics in a variety of applications.

It is this methodology of converting a real-valued data source into a complex signal in order to obtain a set of synchrony features that is novel to our work. The new data representation is then applied as an input to a supervised neural classifier trained to recognize the defective areas from the non-defective ones. To demonstrate the effectiveness of the proposed approach it has been applied to detect and classify internal defects in aircraft composite materials and in particular in a Honeycomb structure containing different inserts placed to simulate some of the most common defective situations in aircraft materials.

\section{The Proposed Approach}

Ultrasonic Inspection uses sound signals at frequencies beyond human hearing (more than $20 \mathrm{KHz}$ ), to estimate some properties of the irradiated material by analysing either the reflected (reflection working modality) or transmitted (transmission working modality) signals. A typical ultrasonic based inspection system consists of several functional units, such as pulser, receiver, transducer, and display devices. A pulser is an electronic device that can produce a high voltage electrical pulse. Driven by the pulser, the transducer generates a high frequency ultrasonic energy. The sound energy is introduced into and propagates through the materials in the form of waves. In the transmission modality the receiver is placed on the opposite side of the material from the pulser whereas in the reflection modality the pulser and the receiver are placed on the same side of the material. Inspection devices can be or not be in contact with the material. In the latter case a liquid (couplant) is used to facilitate the transmission of ultrasonic vibrations from the transducer to the test surface. Ultrasonic data can be collected and displayed in a number of different formats. The three most common formats are known in the Non Destructive Testing (NDT) world as A-scan, B-scan and C-scan presentations. Each presentation mode provides a different way of looking at and evaluating the region of material being inspected. In this paper the analysis of ultrasonic data acquired from the reflection working modality and A-Scan representation is reported. This means that for each 


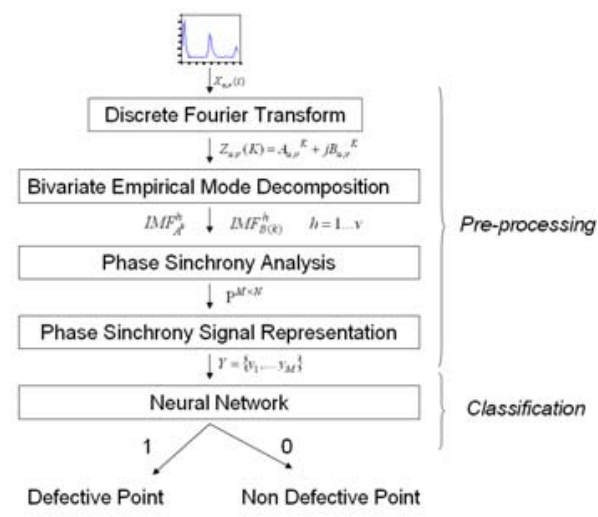

Fig. 1. Scheme of the procedure involved in the proposed approach

point of the inspecting material we have a continuous signal that represents the amount of received ultrasonic energy as a function of time.

The temporal evolution of the ultrasound signal $x_{u, v}(t)$ relative to the position $(u, v)$ onto the plane associated to the upper surface of the material to be inspected is the input to the core of proposed approach that consists of two main steps: the pre-processing of the data in order to emphasize the differences between signals relative to the same class and its subsequent neural classification. The proposed pre-processing step includes multiple operative phases that are schematized in figure 1] In the pre-processing phase, each ultrasound signal $x_{u, v}(t)$ is, firstly, represented in term of its spectral components by using the discrete Fourier transform (DFT). In this way the new signal representation $Z_{u, v}(k)=A_{u, v}(k)+j B_{u, v}(k)$ lies in the field of complex numbers and it is well suited for the next part of the analysis. The signal $Z_{u, v}(k)$ is decomposed into a set of complex IMFs, $\gamma_{i}(z)$ where $i=1, \ldots, M$, by a applying a complex extension of EMD, bivariate EMD [18. The real and imaginary parts of the decomposition, $\Re\left\{\gamma_{i}(k)\right\}$ and $\Im\left\{\gamma_{i}(k)\right\}$, denote the IMFs for $A_{u, v}(k)$ and $B_{u, v}(k)$ respectively. By construction, the phase information for each IMF is well defined at each instant $k$ and facilitates a highly localised comparison between the phase information of $A_{u, v}(k)$ and $B_{u, v}(k)$ [2]. The degree of phase synchrony, the temporal locking of phase information, between $\Re\left\{\gamma_{i}(k)\right\}$ and $\Im\left\{\gamma_{i}(k)\right\}$ is then determined to characterise the dynamics of $Z_{u, v}(k)$. The information is represented by a matrix $\rho_{u, v}(f, k)$ which denotes the synchrony at index $k$ and frequency $f$.

The feature vector $Y_{u, v}(f)=y_{1}, y_{2}, \ldots y_{M}$ relative to the considered $x_{u, v}(t)$ ultrasound signal is finally built by considering the integral of the phase synchrony values for each frequency bin, that is $Y_{u, v}(f)=\int_{0}^{K} \rho_{u, v}(f, k) d k$. The feature vector $Y$ is, finally, normalized (zero mean and unit standard deviation) and it is given as input to a neural classifier trained with a back propagation algorithm to recognize defective from non defective areas. 
The following sub-sections will describe the bivariate empirical mode decomposition strategy and the following phase synchrony methodology.

The EMD Algorithm. Empirical mode decomposition [16] is a data driven time-frequency technique which adaptively decomposes a signal, by means of a process called the sifting algorithm, into a finite set of AM/FM modulated components. These components, called "intrinsic mode functions" (IMFs), represent the oscillation modes embedded in the data. By definition, an IMF is a function for which the number of extrema and the number of zero crossings differ by at most one, and the mean of the two envelopes associated with the local maxima and local minima is approximately zero. The EMD algorithm decomposes the signal $x(t)$ as

$$
x(t)=\sum_{i=1}^{M} C_{i}(t)+r(t)
$$

where $C_{i}(t), i=1, \ldots, M$, are the IMFs and $r(t)$ is the residual. The first IMF is obtained as follows [16.

1. Let $\tilde{x}(t)=x(t)$;

2. Identify all local maxima and minima of $\tilde{x}(t)$;

3. Find an "envelope," $e_{\min }(t)$ (resp. $\left.e_{\max }(t)\right)$ that interpolates all local minima (resp. maxima);

4. Extract the "detail," $d(t)=\tilde{x}(t)-(1 / 2)\left(e_{\min }(t)+e_{\max }(t)\right)$;

5. Let $\tilde{x}(t)=d(t)$ and go to step 2); repeat until $d(t)$ becomes an IMF.

Once the first IMF is obtained, the procedure is applied iteratively to the residual $r(t)=x(t)-d(t)$ to obtain all the IMFs. The extracted components satisfy so called monocomponent criteria and the Hilbert transform can be applied to each IMF separately. This way, it is possible to generate analytic signals, having an IMF as the real part and its Hilbert transform as the imaginary part, that is $x+j \mathcal{H}(x)$ where $\mathcal{H}(\cdot)$ is the Hilbert transform operator. Equation (11) can therefore be augmented to its analytic form given by

$$
X(t)=\sum_{i=1}^{M} a_{i}(t) \cdot e^{j \theta_{i}(t)}
$$

where the trend $r(t)$ is purposely omitted, due to its overwhelming power and lack of oscillatory behavior. Observe from (2), that now the time dependent amplitude $a_{i}(t)$ and phase function $\theta_{i}(t)$ can be extracted. By plotting the amplitude $a_{i}(t)$ versus time $t$ and instantaneous frequency $f_{i}(t)=\frac{d \theta_{i}}{d k}[21$, a timefrequency-amplitude representation of the entire signal is obtained, the so called Hilbert-Huang spectrum (HHS).

Complex Extensions of EMD. In order to obtain a set of $M$ complex/bivariate IMFs, $\gamma_{i}(k), i=1, \ldots, M$, from a complex signal $z(k)$ using bivariate EMD, the following procedure is adopted [18]: 
1. Let $\tilde{z}(k)=z(k)$;

2. To obtain $Q$ signal projections, given by $\left\{p_{\theta_{q}}\right\}_{q=1}^{Q}$, project the complex signal $\tilde{z}(k)$, by using a unit complex number $e^{-\jmath \theta_{q}}$, in the direction of $\theta_{k}$, as

$$
p_{\theta_{q}}=\Re\left(e^{-\jmath \theta_{q}} \tilde{z}(k)\right), q=1, \ldots, Q
$$

where $\Re(\cdot)$ denotes the real part of a complex number, and $\theta_{q}=2 q \pi / Q$;

3. Find the locations $\left\{t_{j}^{q}\right\}_{q=1}^{Q}$ corresponding to the maxima of $\left\{p_{\theta_{q}}\right\}_{q=1}^{Q}$;

4. Interpolate (using spline interpolation) between the maxima points $\left[t_{j}^{q}, \tilde{z}\left(t_{j}^{q}\right)\right]$, to obtain the envelope curves $\left\{e_{\theta_{q}}\right\}_{q=1}^{Q}$;

5. Obtain the mean of all the envelope curves, $m(k)$, and subtract from the input signal, that is, $d(k)=\tilde{z}(k)-m(k)$. Let $\tilde{z}(k)=d(k)$ and go to step 2$)$; repeat until $d(k)$ becomes an IMF.

Similarly to real-valued EMD, once the first IMF is obtained, $\gamma_{1}(k)$, the procedure is applied iteratively to the residual $r(k)=z(k)-d(k)$ to obtain all the IMFs.

Once the IMFs have been obtained, the real and imaginary components can be treated as a two sets of IMFs, $\Re\left\{\gamma_{i}(k)\right\}$ and $\Im\left\{\gamma_{i}(k)\right\}$. The instantaneous amplitudes and phases for each set of IMFs can then be determined.

Phase Synchrony. To measure phase synchrony between $x_{1}$ and $x_{2}$, bivariate EMD is firstly applied to the complex signal $x_{1}+j x_{2}$ [2]. The instantaneous amplitudes for the real and imaginary components of the decomposition, the $i=1 \ldots M$ IMFs at each time instant $k=1 \ldots K$, are denoted by $\Re\left\{a_{i}(k)\right\}$ and $\Im\left\{a_{i}(k)\right\}$ respectively. The instantaneous phase difference between each IMF component is given by $\psi_{i}(k)$. The degree of phase synchrony between $x_{1}$ and $x_{2}$ is given by 2

$$
\phi_{i}(k)=\frac{H_{\max }-H}{H_{\max }}
$$

where $H=-\sum_{n=1}^{N} p_{n} \ln p_{n}$, the Shannon entropy of the distribution of $\psi_{i}(k-$ $\left.\frac{W}{2}: k+\frac{W}{2}\right)$ defined by a window of length $W, \mathrm{~N}$ is the number of bins and $p_{n}$ is the probability of $\psi_{i}\left(k-\frac{W}{2}: k+\frac{W}{2}\right)$ within the $n$th bin [23. The maximum entropy $H_{\max }$ is given by

$$
H_{\max }=.626+0.4 \ln (W-1)
$$

The value of $\phi$ is between 0 and 1, 1 indicating perfect synchrony and 0 a non-synchronous state. An additional step can be incorporated to model simultaneously for component relevance.

$$
\phi_{i}(k)=\left\{\begin{array}{l}
0, \text { if } \Re\left\{a_{i}(k)\right\}<\epsilon P_{r} \\
0, \text { if } \Im\left\{a_{i}(k)\right\}<\epsilon P_{i}
\end{array}\right.
$$

where $P_{r}$ is the power of the original real component (similarly for $P_{i}$ ) and $\epsilon$ is an appropriate threshold. Once the phase synchrony information has been 
estimated, it can be conveniently plotted on a synchrony spectrogram, similar to plots produced in 22. A synchrony spectrogram is essentially a standard time-frequency spectrogram with amplitude information replaced by values for phase coherence. The information can be represented by $\rho(f, k)$, which denotes the phase synchrony at index $k$ and frequency $f$.

\section{Experimental Setup}

To illustrate the effectiveness of the proposed approach a set of ultrasound measurements on a composite material (referred to as the standard in the following) has been considered. The material has a Honeycomb structure with Nomex Core and 48 plies thicknesses. Ultrasonic data were obtained by an ultrasonic reflection technique that uses a single transducer serving as transmitter and receiver $(5 \mathrm{MHz})$. The standard contains artificial defects introduced during the manufacturing process and composed of the following materials: Brass Foil, Pressure Sensitive Tape, Dry Peel Ply, (in the following [A] stands for Tape, [F] for Peel Ply, [B] for Brass). In particular brass inserts were introduced to represent voids and delamination), dry peel ply to represent inclusions by means of reflection techniques and adhesive tape to represent inclusions by means of transmission techniques. For each standard, defects were positioned as reported in figure 2 , The typical insert locations are:

1. Two plies from topside surface for Brass and Pressure Sensitive Tape and five plies for Peel Ply(top)

2. Mid part thickness (mid)

3. One and two plies from backside surface for Brass and Pressure Sensitive Tape and five plies from backside surface for Peel Ply (bottom)

The data set for the considered standard consists of 193x181 spatial samples. Each spatial sample $x_{u, v}(t)$ consists of 77 measurements of the received signal so that the whole data set consists of a matrix sized $193 \times 181 \times 77$. In figure 3 ,

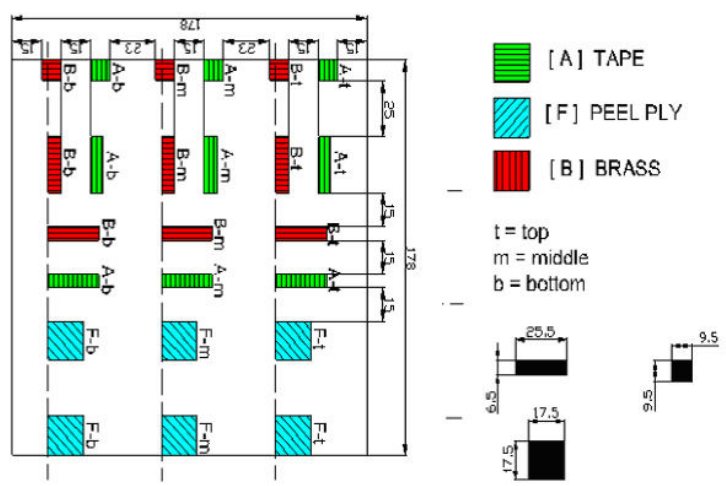

Fig. 2. Defect Scheme 

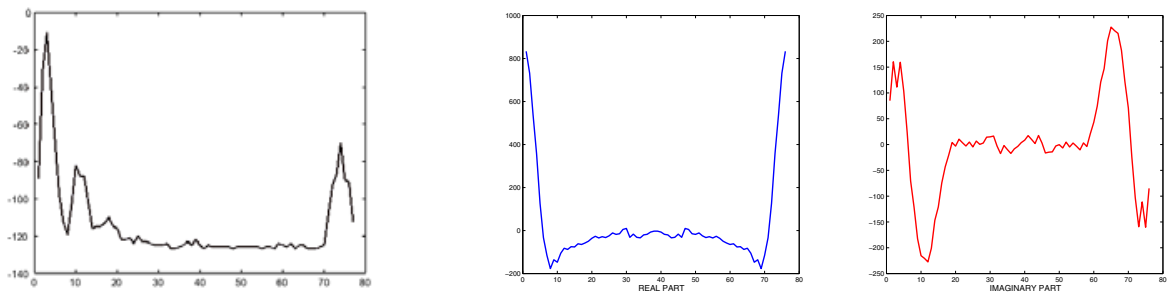

Fig. 3. On the left, an ultrasound signal in A-scan representation is reported. The central and left parts of the figure shows its complex representation after DFT computation.
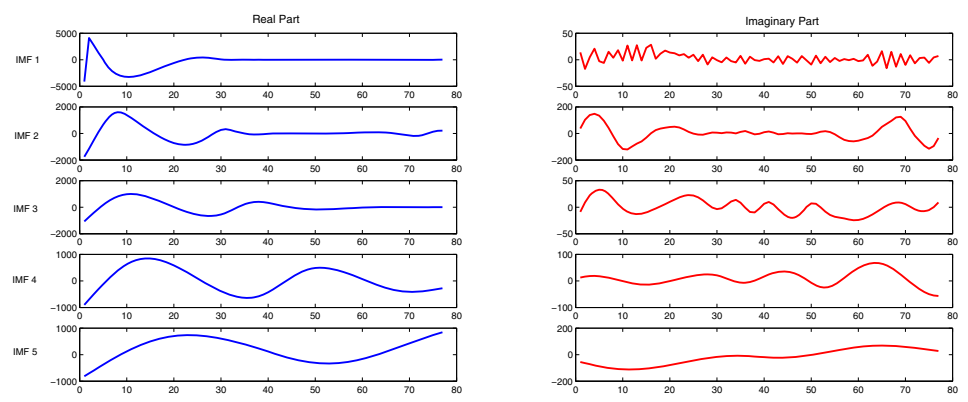

Fig. 4. The EMD decomposition of the DFT of an ultrasound signal: the IMFs relative to the real part of the ultrasound signal are plotted in blue, those relative to the imaginary part are plotted in red

a spatial sample $x_{u, v}(t)$ (i.e. an ultrasound signal in A-scan representation) and its complex representation after DFT computation are shown (real part is plotted in blue, imaginary part in red).

For each input signal, firstly, the DFT was computed and then a complex signal $Z_{u, v}(k)=A_{u, v}(k)+j B_{u, v}(k)$ of length 77 was obtained. The BEMD algorithm was then applied to this complex signal to produce a set of complex IMFs, $\gamma_{i}(k)$. The real part of this decomposition is shown in blue in figure 4, which denotes the IMFs obtained for the real part $A_{u, v}(k)$, whereas the imaginary component of the IMFs, those representing $B_{u, v}(k)$, are shown in red.

The phase synchrony matrix $\rho$ of the IMFs computed for each complex signa 1 associated with the initial ultrasound signal was then computed obtaining a $250 \times 66$ sized matrix for each signal.

Finally the feature vector $Y$ was computed as described in the above section and then a 250 long vector was associated to each ultrasound signal.

${ }^{1}$ In many instances, the IMFs were computed by averaging the decomposition of $Z_{u, v}(k)+v$ where $v$ denotes complex white Gaussian noise of variance smaller than that of $Z_{u, v}(k)$. This enables the embedded data modes to be detected more accurately. For more information on noise assisted EMD, the reader is referred to 3 . 


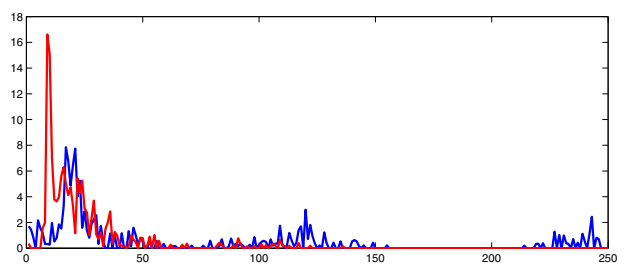

Fig. 5. The feature vector obtained (length 250) for two different signals belonging to a defective area (in blue) and non defective area (in red) respectively

In figure 5 the feature vectors $Y$ for a non defective point (red line) and for a defective point (blue line) are shown.

The feature vectors $Y$ containing phase synchrony patterns were finally supplied as the input to a supervised neural classifier that, for each vector gives a pair of values indicating the class of the considered point in the material. In particular the considered neural network is a 3 layers Back Propagation network with 80 hidden neurons (tansig activation functions) and 2 output neurons (linear activation functions). Defective points are associated with the output values $[1,-1]$ whereas, for non defective points, $[-1,1]$ is the expected output of the neural network. The net has been preliminarily trained using 407 point (207 belonging to defective areas and 200 belonging to non defective areas). In figure 6 the points selected to train the classifier are indicated (white pixels indicate non defective points, black pixels indicate defective points). As reported, the training points have been chosen from a subset of defective areas in order to evaluate the capability of the proposed approach to generalize knowledge on unseen defects.

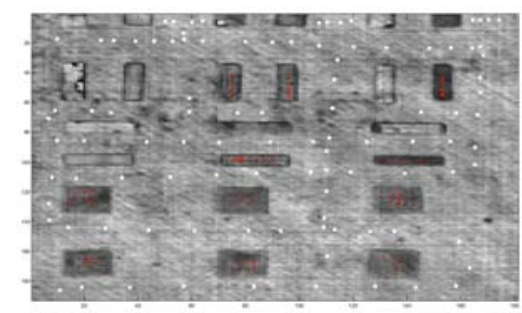

Fig. 6. The points used to train the neural classifier

\section{Experimental Results}

In this section the capability of the proposed framework to automatically detect defective areas in composite materials will be demonstrated and a comparison with standard techniques will be also provided. In particular three experiments have been carried out: 

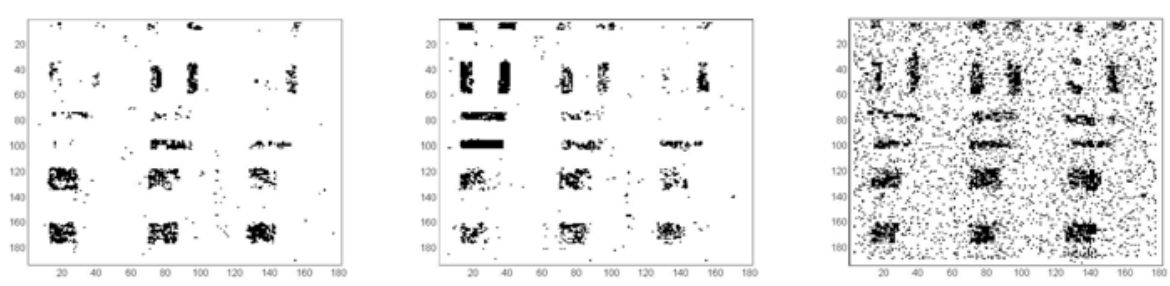

Fig. 7. Defective points detection by supplying as input to the neural network the signal acquired by the receiver (on the left), the wavelet coefficients (in the center) and the synchronization scores (on the right) extracted as described in section 2.

- the ultrasonic signals without any preprocessing have been classified by the neural network (in particular the 77 available samples for each point are given as input to the net after the normalization in the range $[-1,1]))$;

- a classical pre-processing approach based on wavelet decomposition has been applied to the ultrasonic signal and then provided to the classifier (the DB3 family were used and the decomposition was carried out until level 3 . The resulting 92 coefficients were then used to represent the ultrasound signal).

- the proposed preprocessing with EMD has been applied as described in the previous section and then the obtained feature vectors have been classified.

It should be noted that, in order to compare the effects that the different preprocessing techniques have on the final results, in all the three experiments, we have used the same training set, the same neural network and we have applied the same post-processing spatial filtering.

In figure 7 the results obtained in the three experiments are shown. In this figure we report the results obtained by the classifier using the same threshold to separate the defect and sound areas.

Figure 7 demonstrates that the wavelet pre-processing increases segmentation performance with respect the case of no pre-processing but it's clear that only the analysis of the synchrony based on complex extension of EMD and performed on DFT coefficients is able to reveal all the defective areas. At the same time, the EMD based approach, erroneously classified as defective a number of non defective points (false positive occurrences) and, moreover, some holes remained in the defective areas (false negative occurrences).

To partially overcome this problem a post-processing filtering has been applied on each of the above segmented images. In particular, dilation and erosion operations have been performed on the images by using the same $3 \times 3$ square structuring element [15]. After this step the segmentation results in the three considered cases became respectively those shown in figure 8 .

The post-processing step both eliminated most of the false positive occurrences and filled most of the holes in the detected defective areas. After this final step it became much more evident the advantage of using EMD based preprocessing: all the defective regions have been detected and only three small regions have been miss-classified as defective ones. On the contrary the wavelet 

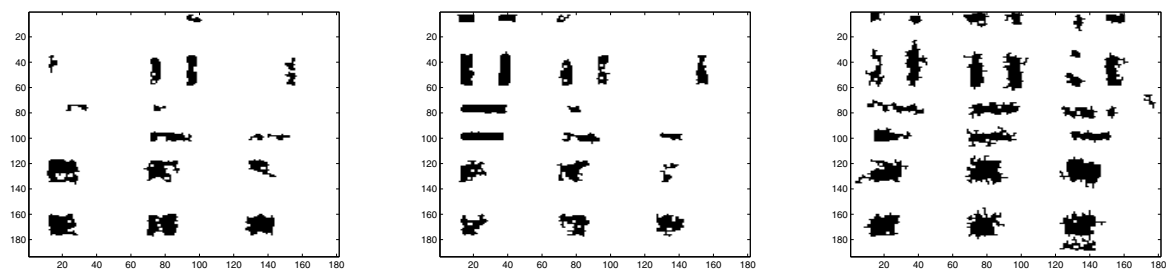

Fig. 8. The regions segmented as defective areas after a spatial filtering based on the connectivity analysis in the case of no preprocessing (on the left), wavelet pre-processing (in the center) and EMD based pre-processing

based pre-processing have not segmented 4 defective regions (practically half of those place on the bottom-side of the standard) whereas in the case of nopreprocessing the miss-detected regions have been much more. On the other side, in the case of wavelet based approach or no-preprocessing, the edge of the detected defective areas are better defined than in case of EMD based preprocessing and this could be very useful in the case where some geometrical description of the detected areas is required. In any case, considering that in the inspection context it is extremely important to detect all the defective areas, even if this could generate some false positive detection, it is possible to conclude that the EMD based pre-processing could be considered the best choice.

\section{References}

1. Boller, C., Chang, F.-K., Fujino, Y.: Encyclopedia of Structural Health Monitoring. John Wiley \& Sons, Chichester (2009)

2. Looney, D., Park, C., Kidmose, P., Ungstrup, M., Mandic, D.P.: Measuring phase synchrony using complex extensions of EMD. In: IEEE/SP 15th Workshop on Statistical Signal Processing, pp. 49-52 (2009)

3. Wu, Z., Huang, N.E.: Ensemble Empirical Mode Decomposition: A noise-assisted data analysis method. Center for Ocean-Land-Atmosphere Studies, vol. 193 (2004)

4. Shah, A.A., Ribakov, Y., Hirose, S.: Nondestructive evaluation of damaged concrete using nonlinear ultrasonics. Materials and Design 30(3), 775-782 (2009)

5. Ruiz-Reyes, N., Vera-Candeas, P., Curpian-Alonso, J., Cuevas-Martýnez, J.C., Blanco-Claraco, J.L.: High-resolution pursuit for detecting flaw echoes close to the material surface in ultrasonic. NDT\&E International 39, 487-492 (2006)

6. Ravanbod, H.: Application of neuro-fuzzy techniques in oil pipeline ultrasonic nondestructive testing. NDT\&E International 38, 643-653 (2005)

7. Bettayeb, F., Rachedi, T., Benbartaoui, H.: An improved automated ultrasonic NDE system by wavelet and neuron networks. Proceedings of Ultrasonics International 42(1-9), 853-858 (2003)

8. Gil Pita, R., Vicen, R., Rosa, M., Jarabo, M.P., Vera, P., Curpian, J.: Ultrasonic flaw detection using radial basis function networks (RBFNs). Proceedings of Ultrasonics International 42(1-9), 361-365 (2003)

9. Margrave, F.W., Rigas, K., Bradley, D.A., Barrowcliffe, P.: The use of neural network in ultrasonic flaw detection. Measurement 25, 143-154 (1999) 
10. Nath, S.K., Balasubramaniam, K., Krishnamurthy, C.V., Narayana, B.H.: An Ultrasonic Time of Flight Diffraction Technique for Characterization of SurfaceBreaking Inclined Cracks. Materials Evaluation 67(2), 141-148 (2009)

11. Rizzo, P., Bartoli, I., Marzani, A., Lanza di Scalea, F.: Defect Classification in Pipes by Neural Networks Using Multiple Guided Ultrasonic Wave Features Extracted After Wavelet Processing. J. Pressure Vessel Technol. 127(3), 294-303 (2005)

12. Wagle, S., Katoa, H.: Ultrasonic wave intensity reflected from fretting fatigue cracks at bolt joints of aluminum alloy plates. NDT\&E Int. 42(8), 690-695 (2009)

13. Meksen, T.M., Boudraa, B., Boudraa, M.: Defects Clustering using Kohonen Networks during Ultrasonic Inspection. IAENG International Journal of Computer Science 36(3) (2009) (online publication)

14. D’Orazio, T., Leo, M., Distante, A., Guaragnella, C., Pianese, V., Cavaccini, G.: Automatic Ultrasonic Inspection for Internal Defect Detection in Composite Materials. Independent NonDestructive Testing and Evaluation 41(2), 145-154 (2008)

15. Gonzales, R.C., Woods, R.E.: Digital Image Processing. Prentice-Hall, Englewood Cliffs (2008)

16. Huang, N.E., Shen, Z., Long, S.R., Wu, M.L., Shih, H.H., Quanan, Z., Yen, N.C., Tung, C.C., Liu, H.H.: The empirical mode decomposition and the Hilbert spectrum for nonlinear and non-stationary time series analysis. Proceedings of the Royal Society A 454, 903-995 (1998)

17. Tanaka, T., Mandic, D.P.: Complex Empirical Mode Decomposition. IEEE Signal Processing Letters 14(2), 101-104 (2007)

18. Rilling, G., Flandrin, P., Goncalves, P., Lilly, J.M.: Bivariate Empirical Mode Decomposition. IEEE Signal Processing Letters 14(12), 936-939 (2007)

19. Umair Bin Altaf, M., Gautama, T., Tanaka, T., Mandic, D.P.: Rotation Invariant Complex Empirical Mode Decomposition. In: Proceedings of the Int. Conf. on Acoustics, Speech and Signal Processing (ICASSP), vol. 3, pp. 1009-1012 (2007)

20. Looney, D., Mandic, D.: Multi-Scale Image Fusion using Complex Extensions of EMD. IEEE Transactions on Signal Processing 57(4), 1626-1630 (2009)

21. Cohen, L.: Instantaneous anything. In: Proc. of the IEEE International Conference on Acoustics, Speech and Signal Processing (ICASSP), vol. 5, pp. 105-108 (1993)

22. Sweeny-Reed, C.M., Nasuto, S.J.: A novel approach to the detection of synchronisation in EEG based on empirical mode decomposition. Journal of Computational Neuroscience 23(1), 79-111 (2007)

23. Tass, P., et al.: Detection of n:m Phase Locking from Noisy Data: Application to Magnetoencephalography. Physical Rev. Letters 81(15), 3291-3294 (1998) 